\title{
Sparse Object Representations by Digital Distance Functions
}

\author{
Robin Strand \\ Centre for Image Analysis, Uppsala University, Sweden \\ robin@cb.uu.se
}

\begin{abstract}
In this paper, some methods for representing objects using path-based distances are considered. The representations can be used as anchor points when extracting medial representations of the objects. The distance transform (DT) is obtained by labeling each object element with the distance to the background. By local operations on the DT, different sets of anchor points can be obtained. We present two different methods based on local operations and prove that the representations are reversible, when this is the case. The methods are defined for weighted distances based on neighborhood sequences, which includes for example the well known cityblock and chessboard distances.
\end{abstract}

\section{Introduction}

The medial axis introduced in 3 is an important and often used concept in image processing. The basic idea is to represent the object with its centerline. When applied to digital images, the set of centers of maximal balls (CMBs) is often used when generating a medial axis to guarantee that the original object can be reconstructed. A ball in an object $X$ is maximal if it is not contained in any other ball in $X$. The original object can be recovered from the set of centers of maximal balls (CMBs) together with the corresponding radii. We say that such a representation is reversible.

In this paper, we focus on distance functions defined as the minimal cost-path between points. With such path-based distance functions, the distance between two points is calculated by counting the number of (weighted) steps needed to go from one point to the other. We distinguish these digital distance functions from the Euclidean metric which is not discrete in this sense. The simplest digital distance functions are the city-block and chessboard distance functions. We consider distance functions defined using both weights (weighted distances) and neighborhood sequences (distances based on neighborhood sequences or nsdistances for short). For the simple distance functions the CMBs appear as local maxima in the distance transform (DT) and are thus easy and fast to extract. For CMBs in the general case with weighted distances or ns-distances, a look-up table is needed in general. In this paper, we present and analyze some natural generalizations of local maxima for weighted ns-distances that are fast and efficient to compute. 
Many authors have considered the local-maxima approach, see, e.g., 131818 . For weighted distances, the local maxima in the DT are the points that do not propagate distance information to neighboring grid points when computing the DT. When non-unit weights or a neighborhood sequence of length $>1$ is used, there are local maxima that are not CMBs. One approach to overcome this problem is to relabel the distance values, see 114. Relabeling is not enough for all distance functions and another approach is to use look-up tables to extract the set of CMBs, see, e.g., 1441211]. Another issue is that the set of local maxima and the points that do not propagate distance information when computing the DT are not always equal.

In this paper, some methods for object representation using local operations for weighted ns-distances will be examined. We consider the following representations:

- Maximal path-points: The points that do not propagate distance information when computing the DT.

- Local B-maxima: When a neighborhood sequence is used to define the distance, the size of the neighborhood at a point $\mathbf{p}$ is given by the neighborhood that is used when computing the DT, can be used to check for local maxima, this is the local B-maxima.

- Local $B^{*}$-maxima: Similar to the local B-maxima, but the size of the neighborhood is given by another element in the neighborhood sequence. Local $B^{*}$-maxima was introduced for ns-distances in [8].

- Reducing the number of points in a reversible representation: By removing superfluous points in a reversible representation, a sparse representation is obtained that is still reversible.

In a previous paper, [15], we found that maximal path-points are not well-suited for representing objects for some distance functions. In this paper, we prove that the set $M P$ of maximal path-points can be computed efficiently by a local approach. We also give an alternative method that can be used also for nsdistances. Some of the results on maximal path-points can also be found in [14].

CMBs and local maxima in DTs using the weighted distance have been used for, e.g., skeletonization algorithms [18 2], object decomposition [17], and resolution pyramids 6]. Local maxima in DTs obtained using ns-distances [8] have also been considered in applications such as discrete shading 9] and normal approximation [10].

\section{Distance Functions}

In this section, some definitions and notions found in, e.g., [141516] are given. Two grid points $\mathbf{p}_{1}=\left(x_{1}, y_{1}\right), \mathbf{p}_{2}=\left(x_{2}, y_{2}\right) \in \mathbb{Z}^{2}$ are $r$-neighbors, $r \in\{1,2\}$, if

$$
\begin{aligned}
\left|x_{1}-x_{2}\right|+\left|y_{1}-y_{2}\right| & \leq r \text { and } \\
\max \left\{\left|x_{1}-x_{2}\right|,\left|y_{1}-y_{2}\right|\right\} & =1 .
\end{aligned}
$$


The points $\mathbf{p}_{1}, \mathbf{p}_{2}$ are adjacent if $\mathbf{p}_{1}$ and $\mathbf{p}_{2}$ are $r$-neighbors for some $r$. Two $r$ neighbors such that the equality in (1) is attained are called strict $r$-neighbors.

Using the notion of 1- and 2-neighbors above, the city-block and chessboard distance functions can be defined. We will consider two ways of generalizing these distance functions by using weights and/or neighborhood sequences.

A ns $B$ is a sequence $B=(b(i))_{i=1}^{\infty}$, where each $b(i)$ denotes a neighborhood relation in $\mathbb{Z}^{2}$. If $B$ is periodic, i.e., if for some fixed strictly positive $l \in \mathbb{Z}_{+}$, $b(i)=b(i+l)$ is valid for all $i \in \mathbb{Z}_{+}$, then we write $B=(b(1), b(2), \ldots, b(l))$.

The following notation is used for the number of $1: \mathrm{s}$ and $2: \mathrm{s}$ in the ns up to position $k$.

$$
\mathbf{1}_{B}^{k}=|\{i: b(i)=1,1 \leq i \leq k\}| \text { and } \mathbf{2}_{B}^{k}=|\{i: b(i)=2,1 \leq i \leq k\}| .
$$

A path $\mathcal{P}_{\mathbf{p}, \mathbf{q}}=\left\langle\mathbf{p}=\mathbf{p}_{0}, \mathbf{p}_{1}, \ldots, \mathbf{p}_{n}=\mathbf{q}\right\rangle$ of length $n$ with start point $\mathbf{p}_{0}$ and end point $\mathbf{p}_{n}$, is a sequence $\mathbf{p}_{0}, \mathbf{p}_{1}, \ldots, \mathbf{p}_{n}$ of adjacent grid points. A path is a $B$-path of length $n$ if, for all $i \in\{1,2, \ldots, n\}, \mathbf{p}_{i-1}$ and $\mathbf{p}_{i}$ are $b(i)$-neighbors.

Definition 1. Given the ns $B$, the ns-distance $d\left(\mathbf{p}_{0}, \mathbf{p}_{n} ; B\right)$ between the points $\mathbf{p}_{0}$ and $\mathbf{p}_{n}$ is the length of (one of) the shortest B-path $(s)$ between the points.

Let the real numbers $\alpha$ and $\beta$ (the weights) and a $B$-path $\mathcal{P}_{\mathbf{p}, \mathbf{q}}$ of length $n$, where exactly $l(l \leq n)$ pairs of adjacent grid points in the path are strict 2neighbors be given. The cost of the $(\alpha, \beta)$-weighted $B$-path $\mathcal{P}_{\mathbf{p}, \mathbf{q}}$ is $(n-l) \alpha+l \beta$. The $B$-path $\mathcal{P}_{\mathbf{p}, \mathbf{q}}$ between the points $\mathbf{p}_{0}$ and $\mathbf{p}_{n}$ is a minimal cost $(\alpha, \beta)$-weighted $B$-path between the points $\mathbf{p}_{0}$ and $\mathbf{p}_{n}$ if no other $(\alpha, \beta)$-weighted $B$-path between the points has lower cost than the $(\alpha, \beta)$-weighted $B$-path $\mathcal{P}_{\mathbf{p}, \mathbf{q}}$.

Definition 2. Given the ns $B$ and the weights $\alpha, \beta$, the weighted ns-distance $d_{\alpha, \beta}\left(\mathbf{p}_{0}, \mathbf{p}_{n} ; B\right)$ is the cost of (one of) the minimal cost $(\alpha, \beta)$-weighted B-path $(s)$ between the points.

Only weights in the interval $\alpha \leq \beta \leq 2 \alpha$ are considered.

We write $\mathcal{L}\left(\mathcal{P}_{\mathbf{p}, \mathbf{q}}\right)$ to denote the length of the path $\mathcal{P}_{\mathbf{p}, \mathbf{q}}$ and $\mathcal{C}_{\alpha, \beta}\left(\mathcal{P}_{\mathbf{p}, \mathbf{q}}\right)$ to denote the cost of the path $\mathcal{P}_{\mathbf{p}, \mathbf{q}}$. The concatenation of two paths $\mathcal{P}_{\mathbf{p}_{0}, \mathbf{p}_{n}}$ and $\mathcal{Q}_{\mathbf{q}_{0}, \mathbf{q}_{m}}$ such that $\mathbf{p}_{n}$ and $\mathbf{q}_{0}$ are adjacent is $\mathcal{P}_{\mathbf{p}_{0}, \mathbf{p}_{n}} \cdot \mathcal{Q}_{\mathbf{q}_{0}, \mathbf{q}_{m}}=\left\langle\mathbf{p}_{0}, \mathbf{p}_{1}, \ldots, \mathbf{p}_{n}, \mathbf{q}_{0}, \mathbf{q}_{1}, \ldots\right.$, $\left.\mathbf{q}_{m}\right\rangle$.

\section{Weighted ns-Distance and Distance Transforms}

We state now a functional form of the distance between two grid points $(0,0)$ and $(x, y)$ in $\mathbb{Z}^{2}$, where $x \geq y \geq 0$. Observe that by translation-invariance and symmetry, the distance between any two grid points is given by the formula presented in Theorem 1 .

The following theorem is proved in [1416]:

Theorem 1. Let the point $(x, y)$, where $x \geq y \geq 0$, be given. The weighted $n s$-distance between $\mathbf{0}$ and $(x, y)$ is given by

$$
\begin{aligned}
d_{\alpha, \beta}(\mathbf{0},(x, y) ; B) & =(2 k-x-y) \cdot \alpha+(x+y-k) \cdot \beta, \\
\text { where } k & =\min \left\{l \in \mathbb{N}: l \geq x+\max \left(0, y-\mathbf{2}_{B}^{l}\right)\right\} .
\end{aligned}
$$


When computing the distance transform, this is of course done on a finite subset of the grid, the image domain $\mathcal{I} \subset \mathbb{Z}^{2}$. The object, a subset of $\mathcal{I}$ is denoted $X$ and its complement in $\mathcal{I}$ is the background $\bar{X}$. We denote the distance transform for path-based distances with $D T_{\mathcal{C}}$, where the subscript indicates that costs are computed.

Definition 3. The distance transform $D T_{\mathcal{C}}$ of an object $X \subset \mathcal{I}$ is the mapping

$$
\begin{aligned}
D T_{\mathcal{C}}: \mathcal{I} & \rightarrow \mathbb{R}_{0}^{+} \text {defined by } \\
\mathbf{p} & \mapsto d(\mathbf{p}, \bar{X}), \text { where } \\
d(\mathbf{p}, \bar{X}) & =\min _{\mathbf{q} \in \bar{X}}\{d(\mathbf{p}, \mathbf{q})\} .
\end{aligned}
$$

For weighted ns-distances, the size of the neighborhood allowed in each step is determined by the length of the minimal cost-paths (not the cost), so this value is also needed when propagating distance information. We define the transform $D T_{\mathcal{L}}$ that holds the length of a minimal cost-path at each point.

Definition 4. The transform $D T_{\mathcal{L}}$ of an object $X$ is the mapping

$$
\begin{aligned}
D T_{\mathcal{L}}: \mathcal{I} & \rightarrow \mathbb{N} \text { defined by } \\
\mathbf{p} & \mapsto d_{1,1}(\mathbf{p}, \mathbf{q} ; B) \text {, where } \\
\mathbf{q} \text { is such that } d_{\alpha, \beta}(\mathbf{q}, \mathbf{p} ; B) & =d_{\alpha, \beta}(\mathbf{p}, \bar{X} ; B) .
\end{aligned}
$$

In 14, algorithms (proved to give correct results) for computing $D T_{\mathcal{C}}$ and $D T_{\mathcal{L}}$ are given. The transforms $D T_{\mathcal{C}}$ and $D T_{\mathcal{L}}$ are illustrated in Figure 1 In the figures, each grid point is represented by the corresponding Voronoi region (picture element or pixel). The algorithms in 14 can also be used to compute the reverse DT, which is used to obtain the original object from the reversible object representation.

\section{Object Representation by Maximal Path-Points}

When extracting CMBs for weighted distances, there is a correspondence between the points that do not propagate distance information and the local maxima in the DT, [12. We will now define this correspondence also for the distances that are defined by neighborhood sequences. A distance propagating path is a path along which local distance values can be propagated when computing the DT. See Figure 1 .

Definition 5. Given an object grid point $\mathbf{p} \in X$ and a background grid point $\mathbf{q} \in \bar{X}$, the minimal cost $B$-path $\mathcal{P}_{\mathbf{q}, \mathbf{p}}=\left\langle\mathbf{q}=\mathbf{p}_{0}, \mathbf{p}_{1}, \ldots, \mathbf{p}_{n}=\mathbf{p}\right\rangle$ is a distance propagating $B$-path if

(i) $\quad \mathcal{C}_{\alpha, \beta}\left(\left\langle\mathbf{p}_{0}, \ldots, \mathbf{p}_{i}\right\rangle\right)=D T_{\mathcal{C}}\left(\mathbf{p}_{i}\right)$ for all $i$ and

(ii) $\mathbf{p}_{i}, \mathbf{p}_{i+1}$ are $b\left(D T_{\mathcal{L}}\left(\mathbf{p}_{i}\right)+1\right)-$ neighbors for all $i$. 
In an object $X$, the end-point of a distance propagating path of maximal length is called a maximal path-point, defined below. A local $B$-maximum is a local maximum obtained by using the neighborhood that is used to propagate distance information at that point, i.e., the neighborhood defined by $b\left(D T_{\mathcal{L}}(\mathbf{p})+1\right)$ at the point $\mathbf{p}$.

Definition 6. A point $\mathbf{p} \in X$ is called a maximal path-point if there is a $\mathbf{q} \in \bar{X}$ and a path $\mathcal{P}_{\mathbf{q}, \mathbf{p}}=\left\langle\mathbf{q}=\mathbf{p}_{0}, \mathbf{p}_{1}, \ldots, \mathbf{p}_{n}=\mathbf{p}\right\rangle$ such that the following statements both hold:

- $\mathcal{P}_{\mathbf{q}, \mathbf{p}}$ is a distance propagating $B$-path of length $n$ defining $D T_{\mathcal{C}}(\mathbf{p})$ and

- there is no $\mathbf{p}^{\prime}$ such that $\left\langle\mathbf{p}_{0}, \mathbf{p}_{1}, \ldots, \mathbf{p}_{n}=\mathbf{p}, \mathbf{p}^{\prime}\right\rangle$, i.e., $\mathcal{P}_{\mathbf{q}, \mathbf{p}} \cdot\left\langle\mathbf{p}^{\prime}\right\rangle$ is a minimal cost $(\alpha, \beta)$-weighted $B$-path of length $n+1$ such that

$D T_{\mathcal{C}}\left(\mathbf{p}^{\prime}\right)=\mathcal{C}_{\alpha, \beta}\left(\left\langle\mathbf{p}_{0}, \mathbf{p}_{1}, \ldots, \mathbf{p}_{n}=\mathbf{p}, \mathbf{p}^{\prime}\right\rangle\right)$.

Also, the path $\mathcal{P}_{\mathbf{q}, \mathbf{p}}$ is called a maximal path.

We denote the set of maximal path-points by $M P$. It is clear that the set of maximal path-points are the points that do not propagate distance information to neighboring grid points when computing $D T_{\mathcal{C}}$.

Definition 7. Let the ns $B$ and the weights $(\alpha, \beta)$ such that $\alpha \leq \beta \leq 2 \alpha$ be given. A point $\mathbf{p} \in X$ is a local $B$-maximum if for all its 1-neighbors $\mathbf{r}^{i}$ :

$$
D T_{\mathcal{C}}\left(\mathbf{r}^{i}\right)<D T_{\mathcal{C}}(\mathbf{p})+\alpha
$$

and, if $b\left(D T_{\mathcal{L}}(\mathbf{p})+1\right)=2$, for all its strict 2 -neighbors $\mathbf{r}^{j}$ :

$$
D T_{\mathcal{C}}\left(\mathbf{r}^{j}\right)<D T_{\mathcal{C}}(\mathbf{p})+\beta
$$

In Theorem 3. we will prove that under some conditions the set of local $B$ maxima and the set $M P$ are equivalent. To check if a grid point $\mathbf{p}$ is a local $B$-maximum, the size of the neighborhood at $\mathbf{p}$ is used. Since the size of the neighborhood at $\mathbf{p}$ is determined by $D T_{\mathcal{L}}(\mathbf{p})$ and $D T_{\mathcal{L}}$ in its original form is not unique for some objects, we need to put an additional constraint on $D T_{\mathcal{L}}$. This problem is illustrated in the following example.

Example 1. Consider $B=(1,2)$ and $(\alpha, \beta)=(2,3)$. In Figure 1(a) and (b), two minimal cost $(2,3)$-weighted $B$-paths from $\bar{X}$ to the point $\mathbf{p}$ with the distance value 10 are shown. Since they are of different lengths, different neighborhoods are considered at $\mathbf{p}$.

We will prove that, when $D T_{\mathcal{L}}$ satisfies the following definition, then the set of maximal path-points and the set of local $B$-maxima are equivalent.

Definition 8. Given an object $X$, a ns $B$ and weights $\alpha, \beta$, we say that $D T_{\mathcal{L}}$ associated with $D T_{\mathcal{C}}$ holds information about the smallest neighborhoods if it holds information about the minimal cost-path with smallest size of the neighborhood at each point of $X$. 


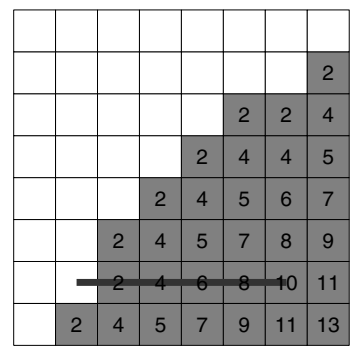

(a)

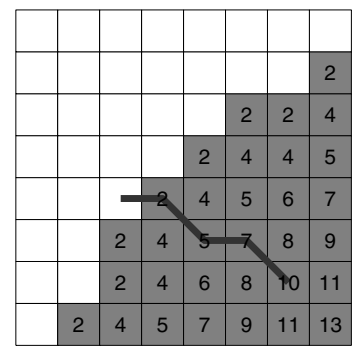

(b)

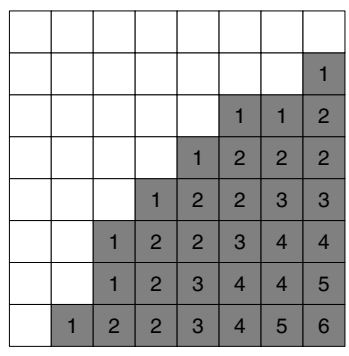

(c)

Fig. 1. (a,b): $D T_{\mathcal{C}}$ with two distance propagating paths overlaid, both containing the point labeled 10, defining different neighborhoods at the point. (c): $D T_{\mathcal{L}}$ With information about the smallest neighborhoods. The ns $B=(1,2)$ and weights $\alpha=2$ and $\beta=3$ are used.

Let the ball obtained by weighted ns-distances with radius $r \in \mathbb{R}^{+}$be $\mathcal{B}(\mathbf{p}, r)=\left\{\mathbf{q}: d_{\alpha, \beta}(\mathbf{p}, \mathbf{q} ; B)<r\right\}$. The main theorem from [15] is that the set $M P$ together with the corresponding distance values is a reversible representation of the object:

Theorem 2. If either

$$
\begin{gathered}
\alpha<\beta \leq 2 \alpha \text { or } \\
\alpha=\beta \text { and }(B=(1) \text { or } B=(2)),
\end{gathered}
$$

then $\forall \mathbf{q} \in X \backslash M P, \exists \mathbf{p} \in M P: \mathbf{q} \in \mathcal{B}\left(\mathbf{p}, D T_{\mathcal{C}}(\mathbf{p})\right)$.

We now give a more efficient way of computing the set $M P$. We will see that, given a point $\mathbf{p}$, it is enough to consider a small neighborhood of that point to determine if it is a maximal point or not. This will be done using the notion of local B-maxima:

Theorem 3. If $D T_{\mathcal{L}}$ holds information about the smallest neighborhoods is used in Definition 7 , then Definition 7 and Definition 6 define the same set of points.

Proof. Let $\mathbf{p} \in X$ be a maximal path-point. Then there is a $\mathbf{q} \in \bar{X}$ and a distance propagating $B$-path $\mathcal{P}_{\mathbf{q}, \mathbf{p}}$ such that any $b\left(\mathcal{L}\left(\mathcal{P}_{\mathbf{q}, \mathbf{p}}\right)+1\right)$-neighbor $\mathbf{r}$ to $\mathbf{p}$ is such that

$$
D T_{\mathcal{C}}(\mathbf{r})<\mathcal{C}_{\alpha, \beta}\left(\mathcal{P}_{\mathbf{q}, \mathbf{p}} \cdot\langle\mathbf{r}\rangle\right)=\mathcal{C}_{\alpha, \beta}\left(\mathcal{P}_{\mathbf{q}, \mathbf{p}}\right)+\omega,
$$

where $\omega$ is $\alpha$ if $\mathbf{p}, \mathbf{r}$ are 1-neighbors and $\beta$ if $\mathbf{p}, \mathbf{r}$ are strict 2-neighbors. This holds for any neighborhood smaller than or equal to $b\left(\mathcal{L}\left(\mathcal{P}_{\mathbf{q}, \mathbf{p}}\right)+1\right)$, so it holds for the smallest neighborhood. This implies that $\mathbf{p}$ is a local $B$-maximum.

If, on the other hand, $\mathbf{p} \in X$ is a local $B$-maximum, then there is a $\mathbf{q} \in \bar{X}$ and a distance propagating $B$-path $\mathcal{P}_{\mathbf{q}, \mathbf{p}}$ with smallest neighborhood at $\mathbf{p}$ such that $D T_{\mathcal{C}}(\mathbf{p})=\mathcal{C}_{\alpha, \beta}\left(\mathcal{P}_{\mathbf{q}, \mathbf{p}}\right)$. For this path, any $b\left(\mathcal{L}\left(\mathcal{P}_{\mathbf{q}, \mathbf{p}}\right)+1\right)$-neighbor $\mathbf{r}$ to $\mathbf{p}$ is such that

$$
D T_{\mathcal{C}}(\mathbf{r})<\mathcal{C}_{\alpha, \beta}\left(\mathcal{P}_{\mathbf{q}, \mathbf{p}} \cdot\langle\mathbf{r}\rangle\right)=\mathcal{C}_{\alpha, \beta}\left(\mathcal{P}_{\mathbf{q}, \mathbf{p}}\right)+\omega
$$


where $\omega$ is $\alpha$ if $\mathbf{p}, \mathbf{r}$ are 1-neighbors and $\beta$ if $\mathbf{p}, \mathbf{r}$ are 2-neighbors. Therefore, $\mathbf{p}$ is also a maximal path-point.

The Algorithm 1 below can be applied to all distance functions presented here. It is a one-scan algorithm. It follows that it is linear in time. Note that for nsdistances (when $(\alpha, \beta)=(1,1)$ ), the set of maximal path-points together with the radii is in general not a reversible representation of $X$.

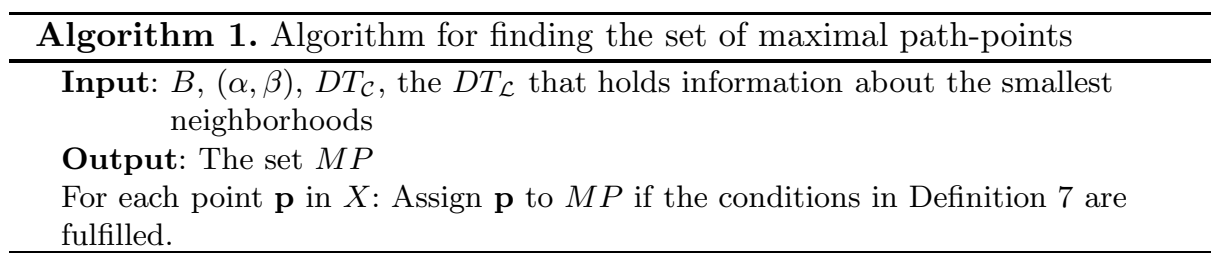

\section{Local $B^{*}$-Maxima}

In the previous section, we used an approach inspired by how algorithms that compute DTs work - the points that do not propagate distance information are stored as the object representation. In this section, an approach similar to the local $B$-maxima will be used. A point $\mathbf{p}$ in an object $X$ is a local $B^{*}$-maximum if it satisfies the following definition:

Definition 9. Let the ns $B$ and the weights $(\alpha, \beta)$ such that $\alpha \leq \beta \leq 2 \alpha$ be given. A point $\mathbf{p} \in X$ is is a local $B^{*}$-maximum if for all its 1-neighbors $\mathbf{r}^{i}$ :

$$
D T_{\mathcal{C}}\left(\mathbf{r}^{i}\right)<D T_{\mathcal{C}}(\mathbf{p})+\alpha
$$

and, if $b\left(D T_{\mathcal{L}}(\mathbf{p})\right)=2$, for all its strict 2 -neighbors $\mathbf{r}^{j}$ :

$$
D T_{\mathcal{C}}\left(\mathbf{r}^{j}\right)<D T_{\mathcal{C}}(\mathbf{p})+\beta .
$$

Note that the only difference between Definition 7 and 9 is that another neighborhood is used to decide when strict 2-neighbors are allowed for finding local maxima. Opposed to the local $B$-maxima, the local $B^{*}$-maxima can be used to derive reversible representations for ns-distances.

Lemma 1. Let $\mathcal{P}_{\mathbf{p}, \mathbf{q}}$ be a minimal cost-path between $\mathbf{p}$ and $\mathbf{q}$. If $\mathbf{2}_{B}^{\mathcal{L}\left(\mathcal{P}_{\mathbf{p}, \mathbf{q}}\right)}>\mathbf{2}_{\mathcal{P}_{\mathbf{p}, \mathbf{q}}}$ and $\mathbf{q}$ and $\mathbf{r}$ are 2 -neighbors, then

$$
d_{\alpha, \beta}(\mathbf{p}, \mathbf{r} ; B) \leq d_{\alpha, \beta}(\mathbf{p}, \mathbf{q} ; B)+\beta .
$$

Proof. Since $\mathbf{2}_{B}^{\mathcal{L}\left(\mathcal{P}_{\mathbf{p}, \mathbf{q}}\right)}>\mathbf{2}_{\mathcal{P}_{\mathbf{p}, \mathbf{q}}}$, there is at least one element 2 in the neighborhood sequence that does not correspond to a strict 2 -step in the path $\mathcal{P}_{\mathbf{p}, \mathbf{q}}$. Therefore, there is a point $\mathbf{r}^{\prime}$ that is 1-neighbor with both $\mathbf{q}$ and $\mathbf{r}$ and a minimal cost-path $\mathcal{P}_{\mathbf{p}, \mathbf{r}^{\prime}}$ obtained by swapping a 1 -step in $\mathcal{P}_{\mathbf{p}, \mathbf{q}}$ to a 2 -step. We have

$$
d_{\alpha, \beta}\left(\mathbf{p}, \mathbf{r}^{\prime} ; B\right) \leq d_{\alpha, \beta}(\mathbf{p}, \mathbf{q} ; B)+\beta-\alpha .
$$

Now, by adding a 1 -step to $\mathcal{P}_{\mathbf{p}, \mathbf{r}^{\prime}}$, a $B$-path between $\mathbf{p}$ and $\mathbf{r}$ of $\operatorname{cost} d_{\alpha, \beta}(\mathbf{p}, \mathbf{q} ; B)+$ $\beta$ is obtained. 
Lemma 2. Given two arbitrary points $\mathbf{w}$ and $\mathbf{p}$.

- If $\mathbf{q}$ and $\mathbf{p}$ are 1-neighbors, then

$$
\mathcal{B}(\mathbf{p}, r) \subset \mathcal{B}(\mathbf{q}, r+\alpha)
$$

where $r=d_{\alpha, \beta}(\mathbf{w}, \mathbf{p} ; B)$.

- If $\mathbf{q}$ and $\mathbf{p}$ are strict 2 -neighbors and there is a minimal cost B-path $\mathcal{P}_{\mathbf{w}, \mathbf{p}}$ between the points such that $b\left(\mathcal{L}\left(\mathcal{P}_{\mathbf{w}, \mathbf{p}}\right)\right)=2$. Then

$$
\mathcal{B}(\mathbf{p}, r) \subset \mathcal{B}(\mathbf{q}, r+\beta)
$$

where $r=d_{\alpha, \beta}(\mathbf{w}, \mathbf{p} ; B)$.

Proof. Consider the case when $\mathbf{q}$ and $\mathbf{p}$ are 1-neighbors. Let $\mathbf{r} \in \mathcal{B}(\mathbf{p}, r)$, i.e., $d_{\alpha, \beta}(\mathbf{r}, \mathbf{p} ; B)<d_{\alpha, \beta}(\mathbf{w}, \mathbf{p} ; B)$. We will now prove that, when the conditions in the lemma is fulfilled, $\mathbf{r} \in \mathcal{B}(\mathbf{q}, r+\alpha)$.

By adding a 1-step to the path defining $d_{\alpha, \beta}(\mathbf{r}, \mathbf{p} ; B)$, a $B$-path between $\mathbf{r}$ and $\mathbf{q}$ of length $d_{\alpha, \beta}(\mathbf{r}, \mathbf{q} ; B)+\alpha$ is obtained. Thus,

$$
d_{\alpha, \beta}(\mathbf{r}, \mathbf{q} ; B) \leq d_{\alpha, \beta}(\mathbf{r}, \mathbf{p} ; B)+\alpha<d_{\alpha, \beta}(\mathbf{w}, \mathbf{p} ; B)+\alpha,
$$

so $\mathbf{r} \in \mathcal{B}(\mathbf{q}, r+\alpha)$.

Now we focus on the case when $\mathbf{q}$ and $\mathbf{p}$ are 2-neighbors. Let $\mathbf{r} \in \mathcal{B}(\mathbf{p}, r)$, i.e.,

$$
d_{\alpha, \beta}(\mathbf{r}, \mathbf{p} ; B)<d_{\alpha, \beta}(\mathbf{w}, \mathbf{p} ; B) .
$$

The path $\mathcal{P}_{\mathbf{r}, \mathbf{p}}$ is a minimal cost $B$-path between $\mathbf{r}$ and $\mathbf{p}$. The path $\mathcal{P}_{\mathbf{w}, \mathbf{p}}$ is a minimal cost $B$-path between $\mathbf{w}$ and $\mathbf{p}$ such that $b\left(\mathcal{L}\left(\mathcal{P}_{\mathbf{w}, \mathbf{p}}\right)\right)=2$ by the conditions in the lemma.

$\star$ Case i $\mathcal{L}\left(\mathcal{P}_{\mathbf{r}, \mathbf{p}}\right) \geq \mathcal{L}\left(\mathcal{P}_{\mathbf{w}, \mathbf{p}}\right)$ (not possible when $\alpha=\beta$, since then the pathlength is proportional to the path-cost)

Since $\mathcal{P}_{\mathbf{r}, \mathbf{p}}$ is longer than $\mathcal{P}_{\mathbf{w}, \mathbf{p}}$, but has lower cost, it follows that $\mathbf{2}_{B}^{\mathcal{L}\left(\mathcal{P}_{\mathbf{r}, \mathbf{p}}\right)}>\mathbf{2}_{\mathcal{P}_{\mathbf{r}, \mathbf{p}}}$. Now

$$
\begin{array}{rlc}
d_{\alpha, \beta}(\mathbf{r}, \mathbf{q} ; B) & \leq d_{\alpha, \beta}(\mathbf{r}, \mathbf{p} ; B)+\beta & (\text { by Lemma 1) } \\
& <d_{\alpha, \beta}(\mathbf{w}, \mathbf{p} ; B)+\beta . \quad(\text { by (4) })
\end{array}
$$

Therefore, $\mathbf{r} \in \mathcal{B}(\mathbf{q}, r+\beta)$

$\star$ Case ii $\mathcal{L}\left(\mathcal{P}_{\mathbf{r}, \mathbf{p}}\right)<\mathcal{L}\left(\mathcal{P}_{\mathbf{w}, \mathbf{p}}\right)$

- Case ii-a $\mathcal{L}\left(\mathcal{P}_{\mathbf{r}, \mathbf{p}}\right)=\mathcal{L}\left(\mathcal{P}_{\mathbf{w}, \mathbf{p}}\right)-1$

Since $b\left(\mathcal{L}\left(\mathcal{P}_{\mathbf{w}, \mathbf{p}}\right)\right)=2$

$$
d_{\alpha, \beta}(\mathbf{r}, \mathbf{q} ; B) \leq d_{\alpha, \beta}(\mathbf{r}, \mathbf{p} ; B)+\beta<d_{\alpha, \beta}(\mathbf{w}, \mathbf{p} ; B)+\beta
$$

Therefore, $\mathbf{r} \in \mathcal{B}(\mathbf{q}, r+\beta)$ also in this case.

- Case ii-b $\mathcal{L}\left(\mathcal{P}_{\mathbf{r}, \mathbf{p}}\right) \leq \mathcal{L}\left(\mathcal{P}_{\mathbf{w}, \mathbf{p}}\right)-2$

- Case ii-b-1 $d_{\alpha, \beta}(\mathbf{r}, \mathbf{p} ; B) \leq d_{\alpha, \beta}(\mathbf{w}, \mathbf{p} ; B)-2 \alpha+\beta$

$$
\begin{aligned}
d_{\alpha, \beta}(\mathbf{r}, \mathbf{q} ; B) & \leq d_{\alpha, \beta}(\mathbf{r}, \mathbf{p} ; B)+2 \alpha \quad \text { independent of } B \\
& \leq d_{\alpha, \beta}(\mathbf{w}, \mathbf{p} ; B)+\beta \quad \text { since Case ii-b-1 }
\end{aligned}
$$


Therefore, $\mathbf{r} \in \mathcal{B}(\mathbf{q}, r+\beta)$

- Case ii-b-2 $d_{\alpha, \beta}(\mathbf{r}, \mathbf{p} ; B)>d_{\alpha, \beta}(\mathbf{w}, \mathbf{p} ; B)-2 \alpha+\beta$

In this case, we have $\mathbf{2}_{\mathcal{P}_{\mathbf{r}, \mathbf{p}}}>\mathbf{2}_{\mathcal{P}_{\mathbf{w}, \mathbf{p}}}$ and since $\mathcal{L}\left(\mathcal{P}_{\mathbf{r}, \mathbf{p}}\right)<\mathcal{L}\left(\mathcal{P}_{\mathbf{w}, \mathbf{p}}\right)$, we have that $\mathbf{2}_{B}^{\mathcal{L}\left(\mathcal{P}_{\mathbf{r}, \mathbf{p}}\right)}>\mathbf{2}_{\mathcal{P}_{\mathbf{r}, \mathbf{p}}}$. Using Lemma 1], we get

$$
d_{\alpha, \beta}(\mathbf{r}, \mathbf{q} ; B) \leq d_{\alpha, \beta}(\mathbf{r}, \mathbf{p} ; B)+\beta<d_{\alpha, \beta}(\mathbf{w}, \mathbf{p} ; B)+\beta,
$$

so $\mathbf{r} \in \mathcal{B}(\mathbf{q}, r+\beta)$.

When applied to a $D T_{\mathcal{L}}$ that holds information about the smallest neighborhoods, Lemma 2 proves the following theorem:

Theorem 4. $\forall \mathbf{r}$ in $X$ that is not a local $B^{*}$-maximum, there is a local $B^{*}$ maximum $\mathbf{p}: \mathbf{r} \in \mathcal{B}\left(\mathbf{p}, D T_{\mathcal{C}}(\mathbf{p})\right)$.

It follows that the linear-time Algorithm 2 below can be used to extract reversible representations of an object $X$.

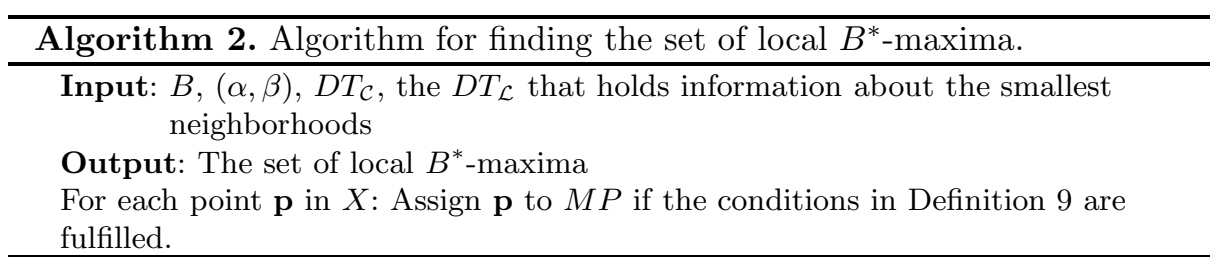

\section{Reducing the Cardinality of Object Representations}

Let the set of points in the object representation be $M R$. In [5], an algorithm that can be used to reduce the set $M R$ is presented. The idea is to iteratively remove the maximal path-points that correspond to balls that are covered by the union of all other balls in $M R$. This is done by, for each $\mathbf{p} \in X$, computing how many balls $\mathcal{B}\left(\mathbf{q}, D T_{\mathcal{C}}(\mathbf{q})\right)$ with center $\mathbf{q} \in M R$ that meet $\mathbf{p}$. If all points in a ball $\mathcal{B}\left(\mathbf{q}, D T_{\mathcal{C}}(\mathbf{q})\right)$ with center $\mathbf{q} \in M R$ meet at least two balls, then $\mathbf{q}$ can be removed from $M R$. Repeating this procedure for increasing distance values gives a representation of $X$ that consist of few points. We call the obtained representation the reduced $M R$. Note that the reduced $M R$ obtained in this way might not be the optimal set, [7.

\section{Examples}

In Figure 2, local $B$-maxima (computed by Algorithm 1), local $B^{*}$-maxima (computed by Algorithm 2), and sets of CMBs are shown using some distance functions. CMBs can be extracted by, e.g., exhaustive search or by look-up tables, 1441211. The object in Figure 2 is not perfectly recovered by the local $B$-maxima for ns-distances, since it is not a reversible representation for nsdistances. 


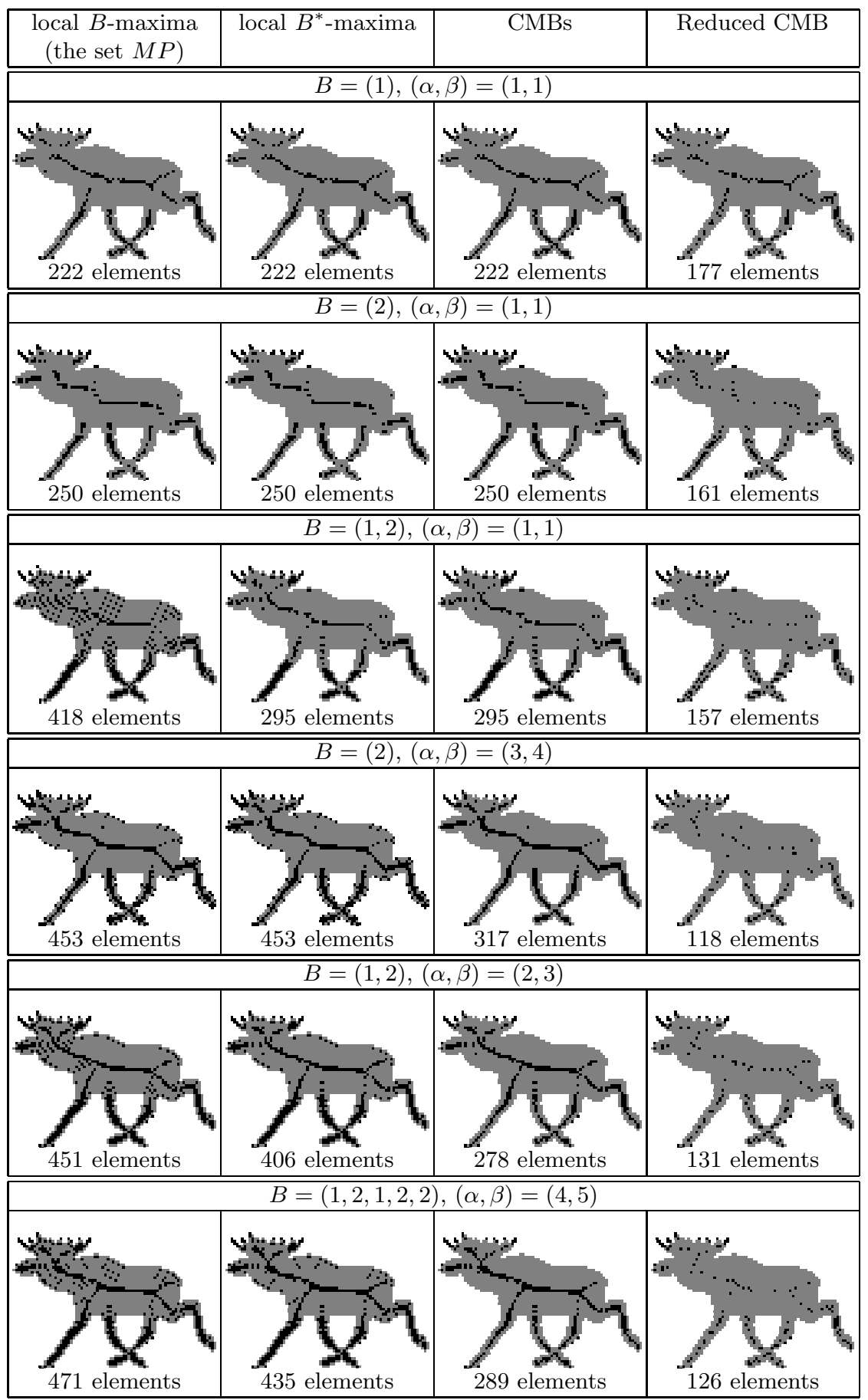

Fig. 2. Object representations for some weighted ns-distances. The object contains 1640 elements. The recovered object is shown for each set of object representations. 


\section{Conclusions and Future Work}

We have presented two methods based on local neighborhoods that can be used for distance functions defined by a neighborhood sequence and weights:

- Maximal path-points (introduced in [15]) defined as the points that do not propagate distance information when computing the DT. We proved that the set of maximal path-points $M P$ can be computed efficiently by finding local $B$-maxima.

- Local $B^{*}$-maxima.

Both methods are fast, since the methods both use local neighborhoods.

If the weights $\alpha$ and $\beta$ are equal, then the method based on maximal pathpoints does not give reversible representations. Note that, when a constant neighborhood sequence is used, the two approaches are equal, see Figure 2.

The object representation based on local $B^{*}$-maxima was used for ns-distances (i.e., without weights) in 8 . The proof in 8 is based on closed balls and the conditions for the non-weighted case are the same as the ones used here.

An interesting observation is that, for ns-distances, the set of local $B^{*}$-maxima is equivalent to the set of CMBs in Figure 2, We are not aware of any verification that this is true in general, but it seems likely, so we leave this as a conjecture.

The quality of the representations for weighted ns-distances using the local operations presented here (local $B$-maxima and local $B^{*}$-maxima) shown in Figure 2 can be improved. It is well-known, [114], that when non-unit weights are used, the distance values in the DT should be relabeled. In [1], it is proved that when using the weights $\alpha=3$ and $\beta=4$ and a constant ns $B=(2)$, then the set of local maxima in a relabeled DT equals the set of CMBs. In [14], object representations obtained by relabeling of general weighted ns-distances combined with local $B$-maxima (i.e., maximal path-points) is described. The number of elements is significantly reduced by this relabeling.

Experimental results have shown that the object representation obtained by relabeling $D T_{\mathcal{C}}$ with weighted ns-distances combined with local $B^{*}$-maxima is very similar to the set of CMBs. We thus have some promising, preliminary result showing that the number of points in the object representation is significantly reduced when relabeling is used. It seems that reducing the object representation in this way does not affect the reversibility. Therefore, we expect our future research to generate strong results on object representations using relabeled DTs.

\section{References}

1. Arcelli, C., Sanniti di Baja, G.: Finding local maxima in a pseudo-Euclidean distance transform. Computer Vision, Graphics, and Image Processing 43, 361-367 (1988)

2. Sanniti di Baja, G., Thiel, E.: Skeletonization algorithm running on path-based distance maps. Image and Vision Computing 14(1), 47-57 (1996) 
3. Blum, H.: A transformation for extracting new descriptors of shape. In: Proceedings of Models for the Perception of Speech and Visual Form, pp. 362-380. MIT Press, Cambridge (1967)

4. Borgefors, G.: Centres of maximal discs in the 5-7-11 distance transform. In: Proceedings of 8th Scandinavian Conference on Image Analysis (SCIA 1993), Troms $\varnothing$, Norway, pp. 105-111 (1993)

5. Borgefors, G., Nyström, I.: Efficient shape representation by minimizing the set of centres of maximal discs/spheres. Pattern Recognition Letters 18, 465-472 (1997)

6. Borgefors, G., Ramella, G., Sanniti di Baja, G., Svensson, S.: On the multiscale representation of 2D and 3D shapes. Graphical Models and Image Processing 61(1), 44-62 (1999)

7. Coeurjolly, D., Hulin, J., Sivignon, I.: Finding a minimum medial axis of a discrete shape is NP-hard. Theoretical Computer Science 406(1-2), 72-79 (2008)

8. Kumar, M.A., Chatterji, B.N., Mukherjee, J., Das, P.P.: Representation of 2D and 3D binary images using medial circles and spheres. International Journal of Pattern Recognition and Artificial Intelligence 10(4), 365-387 (1996)

9. Mukherjee, J., Kumar, M.A., Chatterji, B.N., Das, P.P.: Discrete shading of three-dimensional objects from medial axis transform. Pattern Recognition Letters 20(14), 1533-1544 (1999)

10. Mukherjee, J., Kumar, M.A., Das, P.P., Chatterji, B.N.: Use of medial axis transforms for computing normals at boundary points. Pattern Recognition Letters 23(14), 1649-1656 (2002)

11. Normand, N., Évenou, P.: Medial axis LUT computation for chamfer norms using H-polytopes. In: Coeurjolly, D., Sivignon, I., Tougne, L., Dupont, F. (eds.) DGCI 2008. LNCS, vol. 4992, pp. 189-200. Springer, Heidelberg (2008)

12. Rémy, E., Thiel, E.: Medial axis for chamfer distances: computing look-up tables and neighbourhoods in 2D or 3D. Pattern Recognition Letters 23, 649-661 (2002)

13. Rosenfeld, A., Pfaltz, J.L.: Sequential operations in digital picture processing. Journal of the ACM 13(4), 471-494 (1966)

14. Strand, R.: Distance Functions and Image Processing on Point-Lattices: with focus on the $3 \mathrm{D}$ face- and body-centered cubic grids. Ph.D. thesis, Uppsala University, Sweden (November 2008)

15. Strand, R.: Shape representation with maximal path-points for path-based distances. In: Proceedings of 5th International Symposium on Image and Signal Processing and Analysis (ISPA 2007), Istanbul, Turkey. pp. 397-402 (2007)

16. Strand, R.: Weighted distances based on neighbourhood sequences. Pattern Recognition Letters 28(15), 2029-2036 (2007)

17. Svensson, S., Sanniti di Baja, G.: Using distance transforms to decompose 3D discrete objects. Image and Vision Computing 20(8), 529-540 (2002)

18. Svensson, S., Borgefors, G., Nyström, I.: On reversible skeletonization using anchorpoints from distance transforms. Journal of Visual Communication and Image Representation 10(4), 379-397 (1999) 